\title{
A First Step in Total Quality Management of Nursing Facility Care: Development of an Empirical Causal Model of Structure, Process and Outcome Dimensions
}

\author{
François Sainfort, Ph.D., “ $† \ddagger$, James D. Ramsay, M.A. $\ddagger$, Pedro L. Ferreira, Ph.D.§, \\ and Lassaad Mezghani, M.S.ף
}

\begin{abstract}
†Department of Industrial Engineering, University of Wisconsin-Madison, ¥Center for Health Systems Research and Analysis, University of Wisconsin-Madison, §School of Economics, University of Coimbra, Portugal, $\uparrow$ Ecole Centrale Paris, France
\end{abstract}

While the structure, process, and outcome taxonomy has long been used in the field of health care quality measurement and evaluation, it has not been used in a true causal model which assesses facility level quality. Total quality management and continuous quality improvement call for routinely assessing facility and resident level quality in a causal framework. This paper presents a causal modeling methodology as a more appropriate method for assessing and understanding the inter-relatedness among each of the quality dimensions of Nursing Facility care, and presents how such a causal model directly relates to the notion of continuous quality improvement. The methodology consists of five steps: (1) sample definition and data collection, (2) data reduction through factor analysis, (3) development and testing of a causal model through path analysis, (4) identification of patterns of care through cluster analysis, and (5) integration of the model to both continuous quality improvement and to complex relationships involving quality and organizational variables. The methodology is fully illustrated by using a sample of 104 nursing facilities in Wisconsin in which quality dimensions have been captured through the Quality Assessment Index. The analysis demonstrates that nursing facilities may be substantially benefited by having access to causal linkages which materially affect outcome quality. Management would then have first-hand knowledge of the structural characteristics and the process activities that they may pursue in order to improve outcome quality.

Interest in the determinants of quality in long-term care has increased due primarily to two factors: the

\footnotetext{
* To whom requests for reprints should be addressed at Department of Industrial Engineering, University of Wisconsin-Madison, 1513 University Avenue, Madison, WI 53706.
}

aging of the United States population, and the increasing financial burden of long-term care. Regarding the amount of public money spent on long-term care, nursing facilities (NFs) are the pre-eminent provider type, although most long term care is offered informally by family members in community-based settings. But family members are not always able to offer their loved ones the care they need. In such cases, the family may try and locate the best nursing care for the price. What constitutes the "best" in NF care is a question without a readily available answer. As such, development of valid quality measures and the identification of causal links to characteristics of the care environment have become areas of immense importance to payers, providers, and consumers. However, what is known regarding the determinants of $\mathrm{NF}$ quality is not consistent (1) and thus inhibits informed decisions concerning the quality of NF care. As providers continue to seek ways to improve quality as a means to enhance their competitive position (especially given the odds of universal access form health care reform), knowing what factors cause changes in quality seems imperative.

The international conference on primary care held that any person requiring nursing facility care should be able to enter any certified facility and receive appropriate care, be treated with courtesy, and enjoy continued civil, social, and legal rights (2). While many NFs provide caring environments, many do not (3). The federal regulation of NFs regarding quality assurance is designed to ensure the safety of residents and the adequacy of care. However, in practice, the 
term "adequate" has been interpreted to mean "minimum" acceptable standards (3), standards which are more or less set arbitrarily. Within this interpretation, regulatory agencies tend to rely on review and possible sanctions after the fact to ensure minimal levels of quality.

The purpose of this paper is 2 -fold. By way of an example analysis, it will present a causal modeling methodology as a more appropriate method for assessing and understanding the inter-relatedness among each of the quality dimensions of NF care. Also, it will present how such a Donabedian causal model directly relates to the notion of continuous quality improvement. In this paper, we attempt to shift the quality assessment paradigm from one of retrospectively sanctioning bad quality, to improving quality continuously. We contend that assisting NF administrators in improving quality of care is far more effective and less costly, in the long run, than penalizing those who do not achieve arbitrarily set standards, by better understanding relationships between structure, process, and outcome dimensions of care. The example analysis utilizes quality measures and data from the Quality Assessment Index (QAI) study of NF quality (4).

Ostensibly and as an organizational strategy, it should be possible to improve quality of care without increasing the cost of care, thus leading to a more efficient use of health care resources. Total quality management (TQM) incorporates the notion of continuously improving work processes and therefore the output quality. TQM posits that problems with production quality can be opportunities to improve quality and lower costs due to waste and inefficiently directed work efforts. In this way, TQM suggests that it may in fact save organizations money, in addition to improving their competitive position. Indeed, Deming (5) has suggested that approximately $85 \%$ of all problems with production quality are due to the process and not the worker (5). Continuous quality improvement (CQI) suggests that improvements in quality arise from understanding the causal links in the production process, from inputs to outputs, and from revising processes on the basis of carefully collected data. Processes can and should be continuously monitored and improved. Such an ideal is perfectly aligned with the structure, process, and outcome quality model as proposed by Donabedian $(6,7)$. The structure of the care environment, the processes responsible to improve or stabilize the patient's health status and the resultant outcomes need to be causally linked indicators of quality in order to have an organizationwide appreciation of quality, a central tenet to TQM. Indeed, Jones and Ziegenfuss (8) have recently called for a new look at the Donabedian paradigm with respect to TQM.

In contrast, traditional quality assurance (QA) differs significantly from the basic tenets of TQM: it is not global in its implementation; it does not foster empowerment and participative management among workers; it does not seek to continuously monitor and adjust the production process; and it does not seek to become a principal component of the organization's competitive strategy. The use of ex post facto sanctions is the primary enforcement mechanism of traditional quality assurance. Berwick (9) suggests that the underlying view of traditional QA is that most problems of quality are caused by poor intentions. He puts forth that if one can use deterrence to improve quality, since intentions need to be changed; then one can use reward or punishment to control people who do not care enough to do what they can or what they know is right. It is critical to point out that traditional QA is not inherently a causal model of quality assessment. Therefore, it can contribute only marginally to an organizational assessment of quality within a Donabedian paradigm.

In creating a continuous $\mathrm{NF}$ quality improvement methodology, we suggest that the following three steps are necessary: (a) to define and measure the concept of quality of care using resident level quality indicators which are routinely collected in an attempt to increase use and hold down costs, and which are organized into either process or outcome quality dimensions according to the Donabedian paradigm, (b) to develop an analytic method that should enable clearer understandings of cause and effect relationships among all measures of structure, process, and outcome quality, and (c) to evaluate the feasibility of the system regarding its costs and implementation effects, and what it takes to change some of these factors. The adaptation to and successful application of CQI to health services will not be an easy task (10). In effect, the concept and measurement of quality of care are far more complex than the concept and measurement of the quality of most manufactured products. Data requirements alone may make CQI unfeasible. In addition, knowledge of causal linkages among quality measures and between organizational characteristics and quality measures is sparse.

The following section will review the structureprocess-outcome framework that provides the basis for a causal model of NF quality assessment. We will also briefly review the literature regarding quality/ cost relationships. Next, we will present a generic methodology appropriate to causal analysis. Then we will fully illustrate this causal methodology by an example analysis with QAI data collected on 104 NFs 
in Wisconsin. Finally, we will discuss the results and conclude the paper with a discussion of recommended actions for the continuous improvement of NF quality and further research avenues which we believe need to be explored.

\section{CONCEPTUALIZATION AND RESEARCH MODEL}

\section{Health Care Quality}

Donabedian (7) describes the quality of care "... as that kind of care which is expected to maximize an inclusive measure of patient welfare, after one has taken account of the balance of expected gains and losses that attend the process of care in all its parts" (p. 5). To fully measure quality of care requires a complete accounting of the interplay between and among structural, processes and outcome measures. Briefly, structural measures are those input factors and resources required to provide care. Donabedian (7) refers to them as the "... relatively stable characteristics of the provider, of the tools and resources they have at their disposal, and of the physical and organizational settings in which they work" (p. 81).

Process indicators represent the content, actions, and procedures invoked by the provider in response to the assessed condition of the patient (7). Process quality includes those activities that go on within and between practitioners and patients. For instance, checking medical records for completeness, care plan development, and staff effectiveness training are all items of process quality.

Outcome measures represent the results of the earlier applied structures and processes. Outcomes refer to the "... change in a patient's current or future health status that can be attributed to antecedent health care" (p. 82). Outcome quality includes questions of how the patient fared after the health encounter, whether the patient got "better", how his/her quality of life was affected, including what the extent and nature of the recovery were. Hence, outcome indicators should be represented by both point prevalence and incidence measures.

Thus, quality of care is a multidimensional concept that can be usefully broken down into structure, process, and outcome dimensions which are causally organized. Donabedian (7) notes, "in any system of monitoring [and improving quality], the measurement of outcomes is only the first step in a succession of activities. In order to take corrective action, one must dig back into the processes that led to the unwanted [or desired] outcomes. The identification of the errors in process will, itself, often lead to an examination of the structural features that were responsible for, or contributed to, less than optimal behavior. These necessary activities reaffirm the interconnectedness, the wholeness, of the structure-process-outcome chain. And it is the foundation that any approach to assessment and monitoring must finally rest" (p. 121). This statement implies that structure, process and outcome dimensions of quality are causally linked. If data are appropriately collected to measure the structure, process and outcome aspects of the quality chain, then causal analysis is a logical tool to use in order to elucidate the "interconnectedness" of the chain. Interconnectedness is peculiar to the care setting in which it is applied, as will be further discussed in the next section.

\section{Nursing Facility Quality}

Measuring quality as structure, process, and outcome $(6,7,11)$ is now a well recognized and described causal paradigm. However, the lack of true causal studies of NF quality suggests that the paradigm taxonomy is more often used than the causal framework. As a medical concept, quality has proved to be particularly difficult to measure. Long-term care quality is considered a multidimensional construct subject to the values of those measuring it $(1,12)$. As such, it follows that one could expect to find significant variation regarding both the relationship among various quality measures and between such measures and characteristics of the provider. Indeed, there is a great deal of inconsistency between most studies of NF quality to date (see Davis, (1) for a thorough review). Such variation is largely the result of data limitations. For example, the use of convenience samples has made generalizations difficult, a general lack of causal modeling has exacerbated the inconsistencies among relationships, and an emphasis on cross-sectional data has constrained the use of true outcome measures of quality.

Further complicating matters has been the promulgation of different measurement systems. For example, the Nursing Home Rating Scale (13); the Quality Evaluation System (14); the New York Quality Assessment System (15); the Multiphasic Environmental Assessment Procedure (16); and the Quality Assessment Index (4); although impressive in their design, have not been widely implemented, as they tend to be expensive and tend to rely on numerous, nonroutinely collected data elements at the facility level. As such, they have suffered from small sample sizes making generalizations difficult, and making contin- 
uous quality measurement difficult if not impossible to implement.

Regarding NF quality analysis, we take the term "interconnectedness" to refer to the numerous care goals which define the overall service package of the average NF. NFs typically have several goals of the care they provide, which stem from the very nature of long-term care. Kane and Kane (12) suggest there are nine typical NF goals which include clinical as well as nonclinical aspects. These goals include, but are not limited to: quality of life, psycho-social health, rehabilitation and independence and, of course, the improvement (or stability) in the resident's health status. Complete measurement of quality requires consideration of most, if not all, such care goals. Indeed, many NFs may excel at some of the care goals, even emphasize them, but may not succeed at others. Using the structure, process, and outcome paradigm helps to insure that complete consideration of all aspects of quality are included in an assessment of facility care. Hence several measures of structure, process, and outcome quality are necessary to completely operationalize the NF quality construct. Placing that measurement paradigm in a causal framework insures that the interconnectedness of the entire construct is appropriately considered in the analysis. The result is a more complete view of the relationships among all aspects of quality at both the facility and resident levels.

\section{Quality Versus Cost of Care}

The Donabedian $(7,11)$ framework implies a distinct relationship between the quality of care, the cost of that care, and the risks of receiving that care. In general, these relationships are not linear. Instead, they relate nonlinearly and are best represented by an inverted "U" shaped curve. That is, there is some threshold of medical services, above which the costs and medical risks rise faster than the clinical benefits accrue. Quality is said to be maximal at the point where benefits are maximized and risks and costs are minimized, i.e., at the apex of the benefits curve and necessarily at the minimum of the cost curve.

Expenditures have typically been regarded as essential inputs into the production of quality. It has been conventional wisdom to believe that as expenditures increase, so does quality. However, Nyman (17) explains that most quality-expenditure studies are confounded. He suggests that since expenditures are typically inputs (i.e., in the form of structural components of quality) into the care environment, one would expect to see higher quality with higher expenditures. A consistent link between expenditures and either process or outcome indicators of quality has not yet been established (1).

Expenditure-quality studies have included mainly structural quality indicators with, more recently, process and outcome indicators. Expenditures themselves are often considered structural elements to the care process $(18,19)$. Also, Davis (1) speculates that process and outcome indicators of quality may be independent of expenditures. This paper contends the opposite. In any causal model of quality $(7,11)$, no structural element would be truly independent of elements in subsequent parts of the model. Inconsistency with expenditure-quality studies is in part due to varied operationalizations of quality; but, is also due to the role expenditures have in the production of quality. That is, where in a causal path would expenditures fit in? Most studies do not specify the exact way in which expenditures figure into the production of care (1). Lack of specification can be considered a deficiency in the causal literature regarding the relationship between expenditures and NF quality.

The logic behind the expenditure-quality relationship lies mainly in how expenditures represent direct care. Direct care (assisting in activities of daily living, managing behavior, and communication, etc.) accounts for the bulk of all NF expenditures on care (20). The degree of direct care provided by a given home is typically related to the case mix of the NF. Presumably, a more difficult case mix would require more direct care, that is, more expensive "hands on" nursing care which can potentially require a greater percentage of professional staff. In this sense, heavy care residents are not typically considered "desirable" residents under most current reimbursement systems. Since most reimbursement systems are not case mixsensitive, NFs would get the same reimbursements for light care and heavy care residents, but may have vastly different nursing and administrative work loads associated with each. As a further complication, it is more difficult to create improvement in some heavy care residents, e.g., quadriplegics. The clinical goal of these residents is often maintenance, as opposed to cure $(1,12)$. Hence, expenditures could increase with no obvious change in health status. Without accounting for case mix, and without specifying exactly which expenditures are being referred to, it is difficult to articulate a nonspurious expenditure-quality relationship. For this reason, it is critical to include case mix adjusted measures of direct care in any analysis of $\mathrm{NF}$ quality.

The exact specification and case mix adjustment of expenditures is clearly one source of confusion regarding the NF quality literature. Organizational slack is 
another. Expenditures toward a complex organizational product such as health care, may not always translate directly into increased quality of the product because of organizational slack (1). Organizational slack can be thought of as the degree of excess capacity maintained by the firm in order to assure the reliability of performance (21). Population ecology suggests that the efficiency differences between specialists and generalists arises partly due to the diminished need for excess capacity by specialists. In this sense, generalism is a costly strategy to maintain. The degree to which excess capacity is maintained is related to the rapidity with which the environment is changing. More capacity is required by a more dynamic environment (21). Hence, inconsistency in the cost-quality literature likely arises in part from the notion that NFs with varying environmental constraints may procure varying degrees of excess capacity depending on how they have identified their role in the market; and how they perceive and react to threats in their environment. Organizational slack is an important reason why several measures of structure are needed to completely model the causal relationships between the structure, process, and outcome element of NF quality.

The next section will discuss the proposed methodology for the development of a causal model of NF quality.

\section{METHODOLOGY}

A causal modeling methodology is an appropriate method for assessing and understanding the interrelatedness among the various dimensions of NF care, within a Donabedian framework. However, in order to develop such an empirical model, significant amounts of data and analyses need to be performed. This lead the authors to a five-step methodology: (1) sample definition and data collection, (2) data reduction through factor analysis, (3) development and testing of a causal model through path analysis, (4) identification of patterns of care through cluster analysis, and (5) integration of the model to both continuous quality improvement and to complex relationships involving quality and structural variables.

\section{Step 1: Sample Definition and Data Collection}

First, valid and reliable data regarding the structure, process, and outcome aspects of NF quality of care need to be collected on a population of nursing facilities. If possible, such data should contain both resident level and facility level data. The resident level data should be aggregated at the facility level in order to allow subsequent analyses with the nursing facility as the unit of analysis. Whenever possible, these measures should be adjusted to take into account differences in residents across nursing facilities (such as case mix).

\section{Step 2: Reduction to Underlying Dimensions}

Second, underlying structure, process, and outcome dimensions should be determined in order to reliably and validly define these constructs and in order to reduce the dimensionality of the variables set. The list of variables selected to measure quality can usually be logically classified as structure, process, or outcome. However, we recommend performing a confirmatory factor analysis to examine the hypothesized relationships and identify any unexpected relationships. By performing a factor analysis, the variables group themselves into a smaller number of underlying dimensions that can be interpreted as various global measures of quality of care. Such a reduction enables a more parsimonious evaluation of the causal model. Several factor solutions should be examined so as to choose the most appropriate number of dimensions. A number of analytical criteria can be used to select the number of dimensions, including: the cumulative percentage of total variation explained by the factor solution, both Kaiser's and Jolliffe's rules, and a graphical analysis of the scree graph and the logeigenvalue diagram (22). The final choice of the number of dimensions to be retained, however, is usually based on ease of interpretation and parsimony. Once identified, these dimensions will enable the development of scales and indices that combine the variables into a set of more parsimonious measures (23). Several techniques exist to construct such scales including: multiple regression, the least-squares criterion, Bartlett's criterion, or a summation of variables with high factor loadings $(23,24)$.

\section{Step 3: Development of A Causal Model}

Third, the underlying dimensions of quality should be used to develop a causal model of quality consistent with Donabedian's paradigm. The structure-processoutcome causal model can be tested using path analysis. Path analysis is an appropriate technique to test hypothesized causal models (25-27). Path analysis provides explanations of cause-and-effect relations among variables, based on the observed correlations. It is a specific statistical technique based on row and 
standardized multiple regression (28), and weighted regression with proportion or percentage differences $(29,30)$. The regression coefficients for the standardized predictors are called path coefficients. The path coefficients express the importance of the direct and indirect influence the predictor variables have upon the criterion variable. Comparisons of path coefficients enable tests of direct versus indirect effects of structural measures of quality on outcome measures of quality.

The overall path analysis strategy follows the logic of well-described nonrecursive causal modeling procedures $(25,26)$. First, process variables are regressed over structure variables. Second, outcomes are regressed over structure and process variables. Third, direct and indirect effects of structure variables are assessed using the beta-coefficients of the various regression equations. For example, in a situation with two outcome variables $\mathrm{O}_{1}$ and $\mathrm{O}_{2}$, two process vari-. ables $P_{1}$ and $P_{2}$ and two structure variables $S_{1}$ and $S_{2}$, the direct and indirect effects of $\mathrm{S}_{1}$ on $\mathrm{O}_{2}$ will be computed as follows:

Regression equations with beta-weights:

$\mathrm{O}_{2}=\mathrm{a}+\mathrm{b}_{1} \times \mathrm{P}_{1}+\mathrm{b}_{2} \times \mathrm{P}_{2}+\mathrm{b}_{3} \times \mathrm{S}_{1}+\mathrm{b}_{4} \times \mathrm{S}_{2}$

$\mathrm{P}_{1}=\mathrm{c}+\mathrm{d}_{1} \times \mathrm{S}_{1}+\mathrm{d}_{2} \times \mathrm{S}_{2}$

$P_{2}=a+f_{1} \times S_{1}+f_{2} \times S_{2}$

Estimation of direct and indirect effects of $\mathrm{S}_{1}$ on $\mathrm{O}_{2}$ :

direct effect $=b_{3}$

indirect effect $=b_{1} \times d_{1}+b_{2} \times f_{1}$

Finally, the path coefficients are tested to determine whether or not a path assumed to be zero is actually zero and the path analysis model is re-evaluated without the nonsignificant paths.

\section{Step 4: Identification of Patterns of Care through Cluster Analysis}

It is evident that all nursing facilities are not organized in the same way, nor do they try to deliver the same quality care $(3,4)$. For example, they differ in terms of certification status, ownership status, location, size, staffing, and amenities, etc. Organizational theory suggests that facilities adapt to their operating environments, and economic theory suggests that facilities will enhance quality only when the market environment is competitive. We thus suggest using cluster analysis to identify facility clusters which can be defined according to variables that characterize the market environment, organizational characteristics (ownership, chain status, and facility size), structural features, and process quality. The variables selected for inclusion in the cluster analysis will ultimately depend on the sample of nursing homes used to carry out such a study. For example, in the current study, no market environment characteristics were included since all nursing facilities in the sample were in the same state. Clusters should be clearly distinct in composition, each corresponding to different modes of operation in different environments with different goals and strategies, i.e., different patterns of care. Only when such patterns of care are identified and controlled, can meaningful investigations of specific complex issues (i.e., the relationship between cost and quality) be performed. Independently examining each cluster before combining the results across clusters can provide a more thorough understanding of these issues. In addition, clusters also allow benchmarking of facilities with the highest quality within each cluster, ceteris paribus. Benchmarking done this way is much more informative than benchmarking on a general population of nursing facilities without having accounted for differences in care patterns.

\section{Step 5: Integration of the Model to Total Quality Management}

The final step consists of integrating the results of the causal model, along with the results of the clustering process as well as any investigations of other complex relationships (i.e., quality and cost) into a continuous quality improvement framework.

The current study applied the above methodology to a sample of nursing facilities in Wisconsin. In 1981, the State of Wisconsin engaged in a restructuring of the NF quality assurance process. As part of this new process, Gustafson et al. (4) developed the QAI to measure quality of care in nursing homes. The QAI is a multiattribute utility model that considers seven general areas of care. It is a multidimensional model that incorporates measures of structure, process, and outcome quality. The derivation of the quality variables and its reliability and validity have been tested and reported elsewhere (4).

A total sample of 104 randomly selected nursing facilities in Wisconsin were visited by a team consisting of one registered nurse and one social worker. The team applied the QAI to each of the nursing facilities. In addition, organizational variables were collected in the nursing homes either directly from the facilities or from annual reports available at the Wisconsin State Department of Health and Social Services.

These data provided the basis for developing a causal model and examining the cost versus quality relationship according to the methodology outlined above. The following section describes the results of 
the study and illustrates possible linkages to tenets of continuous quality improvement.

\section{RESULTS}

\section{Step 1: Summary Statistics}

The summary statistics of all variables used in this study are shown in Table 1 . Table 1 lists the organizational characteristics first, and then lists the quality variables collected by the QAI. These quality measures have been classified as one of the three types; as either structure, process, or outcome in accordance with the Donabedian $(6,7)$ paradigm.

\section{Step 2: Reduction to Underlying Subdimensions Through Factor Analysis}

Confirmatory factor analyses were performed on the variables within each of the three quality dimensions. Two decision rules were used to help determine an appropriate number of underlying subdimensions. First, a minimum cumulative percentage of variance explained was set at $60 \%$, and second, a graphical

Table 1

Variables Description and Selected Statistics $(N=104)$




Table 2

Factor Loadings for Structure Variables

\begin{tabular}{lcr}
\hline \multicolumn{1}{c}{ Variables } & $\begin{array}{c}\text { Factor 1 } \\
\text { Staff }\end{array}$ & $\begin{array}{c}\text { Factor } 2 \\
\text { Facility }\end{array}$ \\
\hline - Total nursing staff & 0.878 & -0.049 \\
- Registered nurses & 0.844 & 0.093 \\
- Credentials of nursing staff & 0.545 & 0.363 \\
& & \\
- Facility floor plan & 0.077 & 0.836 \\
- Facility orientation/protective measures & 0.061 & 0.706 \\
\hline
\end{tabular}

analysis of the scree graph (22). Tables 2 through 4 present the loadings for each quality variable on the underlying rotated subdimensions. After examining the different factor solutions for each group, we determined that the structure variables could be grouped into two subdimensions: staff and facility. The twofactor solution for structure variables explains $89.4 \%$ of the total variance. The process quality variables could be grouped into three subdimensions: social care, medical care, and resident influence in care. The three-factor solution for process variables explains $65.7 \%$. Finally, the outcome quality variables could be grouped into three subdimensions: medical outcome, social outcome, and satisfaction. The three-factor solution for outcome variables explains $61.3 \%$ of the total variance. Overall, the factor analysis enabled reduction of the 19 quality variables from the QAI into 8 meaningful underlying subdimensions of quality.

Figure 1 represents all quality variables and the underlying subdimensions in a cause-and-effect diagram (31). Such diagrams illustrate the causal linkages among and between quality measures and dimensions. They are commonly used management tools in ac- cordance with the basic tenets of continuous quality improvement. Each of the eight subdimensions is used to develop a causal model through path analysis.

\section{Step 3: Development of the Causal Model Through Path Analysis}

First, we tested the structure-process-outcome quality model at a more aggregated level by further grouping the dimensions into three overall blocks as suggested by Davis (29). Figure 2 shows the aggregate results of the path analysis. Figure 2 shows both the direct effect as well as the residual effect path estimates (i.e., the standardized regression coefficients) for outcome on process and structure, and for process on structure are shown for each linkage. The indirect relationship of structure and outcome is calculated as the product of the regression coefficients of process on structure (0.186), and of outcome on process $(0.402)$, that is 0.075 . The direct relationship is the regression coefficient of outcome on structure, 0.079 . Since neither are zero, we conclude that there is some direct effect of structure on outcome, as well as some indirect effect. Nevertheless, these coefficients are both close to zero, implying that the magnitude of the effect is small.

Determining whether a path coefficient is significantly different from zero is usually done by comparing the partial correlation to a predetermined threshold, e.g., 0.10 (25). Using this threshold, the direct path from structure to outcome would be nonsignificant. This would lend more support to the hypothesis of a true causal model, where structural characteristics of a nursing home influence the outcome quality only through the process of care.

Table 3

Factor Loadings for Process Variables

\begin{tabular}{|c|c|c|c|}
\hline Variables & $\begin{array}{c}\text { Factor } 1 \\
\text { Social care }\end{array}$ & $\begin{array}{c}\text { Factor } 2 \\
\text { Medical care }\end{array}$ & $\begin{array}{c}\text { Factor } 3 \\
\text { Resident influence }\end{array}$ \\
\hline $\begin{array}{l}\text { - Staff attitudes to resident } \\
\text { - Variety/adequacy of resident } \\
\text { activities }\end{array}$ & $\begin{array}{l}0.792 \\
0.723\end{array}$ & $\begin{array}{r}0.219 \\
-0.061\end{array}$ & $\begin{array}{l}0.049 \\
0.108\end{array}$ \\
\hline - Match of residents to activities & 0.589 & 0.121 & 0.517 \\
\hline $\begin{array}{l}\text { - Staff Effectiveness } \\
\text { - Evaluation/Assessment of } \\
\text { Residents }\end{array}$ & $\begin{array}{r}-0.263 \\
0.320\end{array}$ & $\begin{array}{l}0.793 \\
0.677\end{array}$ & $\begin{array}{r}-0.017 \\
0.059\end{array}$ \\
\hline $\begin{array}{l}\text { - Plan of care development and } \\
\text { implementation }\end{array}$ & 0.345 & 0.624 & 0.366 \\
\hline $\begin{array}{l}\text { - Resident influence in process } \\
\text { of care }\end{array}$ & -0.001 & 0.064 & 0.919 \\
\hline
\end{tabular}


Table 4

Factor Loadings for Outcomes Variables

\begin{tabular}{|c|c|c|c|}
\hline Variables & $\begin{array}{c}\text { Factor } 1 \\
\text { Medical outcome }\end{array}$ & $\begin{array}{c}\text { Factor } 2 \\
\text { Social outcome }\end{array}$ & $\begin{array}{c}\text { Factor } 3 \\
\text { Satisfaction }\end{array}$ \\
\hline $\begin{array}{l}\text { - Resident Awareness } \\
\text { - Resident mood } \\
\text { - Resident physical condition } \\
\text { - Communication between residents } \\
\text { - Continuity of lifestyle } \\
\text { - Complaints } \\
\text { - Violations }\end{array}$ & $\begin{array}{r}0.668 \\
0.672 \\
0.630 \\
0.025 \\
0.003 \\
-0.246 \\
0.190\end{array}$ & $\begin{array}{r}0.524 \\
-0.003 \\
-0.099 \\
0.750 \\
0.743 \\
0.022 \\
-0.294\end{array}$ & $\begin{array}{r}0.052 \\
0.050 \\
-0.382 \\
-0.039 \\
-0.144 \\
0.806 \\
0.768\end{array}$ \\
\hline
\end{tabular}

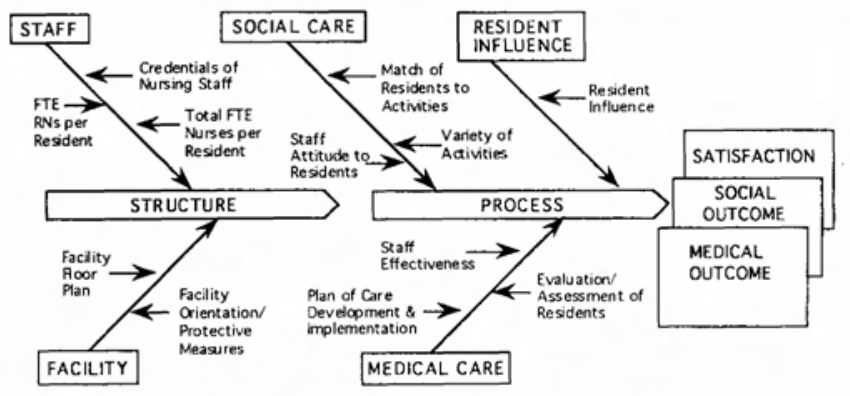

Fig. 1. Cause and Effect Diagram.

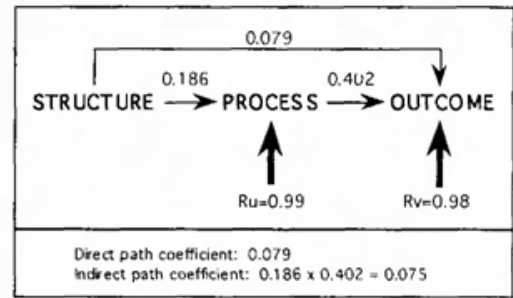

Fig. 2. General Level Path Analysis.

Second, in order to better understand the nature of the causal relationships between all three dimensions of quality, we tested the causal model at a more operational level. That is, using the eight subdimensions of quality of care. The results of the detailed path analysis are shown in Figure 3. In this model, we also divided the third outcome factor, satisfaction into its two components, complaints and violations. This was done in order to gain additional information on what contributes to these outcomes. Only the significant paths are shown in Figure 3. The plain solid arrows represent positive direct paths from structure to process and from process to outcome, along with their path coefficients. The dashed arrows show negative direct paths from structure to outcome, along with the path coefficients. Finally, the double arrows show the correlation among dimensions within the same group and indicate that no causal relationship is assumed.

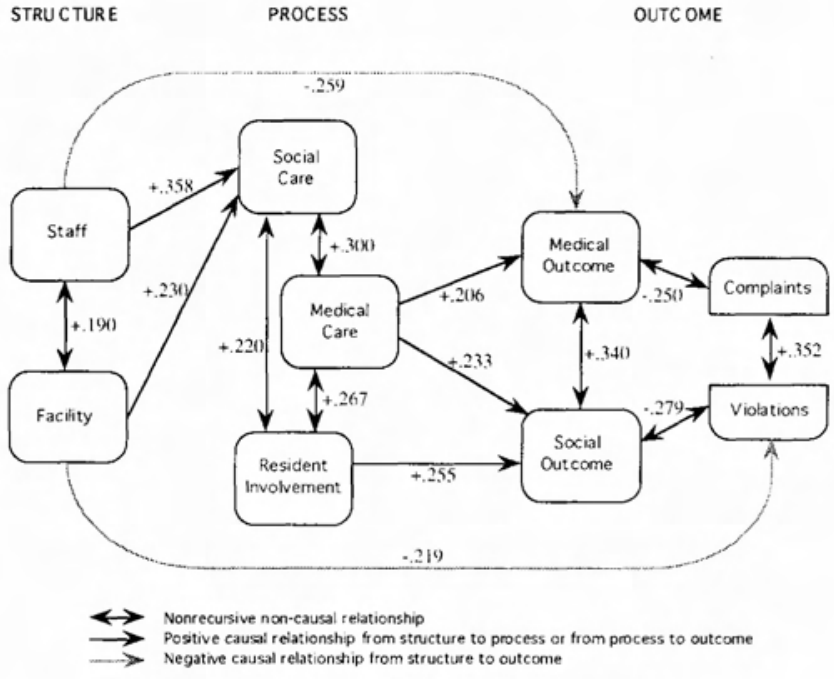

Fig. 3. Detailed Path Analysis.

As can be seen, social care provided in the nursing home is primarily determined by the composition and effectiveness of the staff, the characteristics of the facility, and the involvement of residents in the process of care. Contrary to the original classification, it seems as if social care is in itself an outcome rather than a process variable. The line between the endresult of a process and a so-called "outcome" is often difficult to draw. It is more important to recognize what elements tend to contribute to good social care. In addition, social care processes and medical care processes are strongly correlated, +0.30 .

The only unexpected result noted is that the staff contributes negatively to medical outcome, -0.259 . This counterintuitive causal relationship may reflect the lack of case mix adjustment of the quality variables. However, even without case mix adjustment, it should be noted that the staff positively affects medical outcome indirectly through both social and medical care processes. Medical care is an important predictor of both medical outcome and social outcome. Social care is also such a predictor, but indirectly 
through medical care. Social outcome is explained directly by medical care and resident involvement, and indirectly by social care and its own determinants, that is staff, facility, and resident involvement. Finally, in terms of the organizational outcomes, complaints are directly related to poor medical outcomes, whereas violations are caused directly by poor facility characteristics and are related to poor social outcomes.

\section{Step 4: Identification of Patterns of Care through Cluster Analysis}

Because nursing facilities are organized very differently and might strive for different goals, cluster analysis was necessary to identify patterns of care. The similarity among clusters can be defined in various ways (32). In the current study, the Euclidean distance between facilities was used to judge similarity. Distance was measured in terms of the variables or characteristics selected for the cluster analysis: organizational characteristics, structure, and process dimensions. The greater the distance, the less the similarities.

By looking at the centroids of each cluster (i.e., the stereotype of each pattern of care), it appears that the predominant organizational characteristics in clustering was the certification status of the nursing facility. In our sample there were $27(26.0 \%)$ intermediate care facilities (ICF) and 77 (74.0\%) skilled nursing facilities (SNF). The next most important set of variables that differentiate among clusters, were the process dimension variables. For each certification status, we found two clusters, one with high levels of quality in the process dimensions (ICF group 2 with $N=16$ facilities; and SNF group 2 with $N=30$ facilities) and one with a lower level of quality in the process dimensions (ICF group 1 with $N=6$ facilities; and SNF group 1 with $N=46$ facilities), for a total of four clusters. The ICF group 2 was not significantly different from the SNF group 1 in terms of the process dimensions. We disregarded six other clusters due to a lack of facilities within each of them (three or fewer). The remaining organizational characteristics and structural dimensions did not further differentiate the clusters. It should be noted, however, that a larger sample of nursing facilities would probably have lead to different patterns of delivery of care. Nevertheless, as explained below, the limited differentiation proved to be useful.

\section{Step 5: Integration to Total Quality Management}

To illustrate the potential usefulness of this methodology to TQM, we studied how the clusters per- formed on outcome quality dimensions, as well as costs. We standardized (i.e., to a mean of 0 and standard deviation of 1) all three outcome dimensions; social outcome, medical outcome, and satisfaction. Standardization facilitated comparisons across clusters. All outcome dimensions are in the same direction (the more standard units, the higher the quality). Figure 4 shows the profiles of each cluster which are plotted as average facility per diem (vertical axis) as a function of standard units of each outcome quality dimension (horizontal axis). As one would have expected, both SNF groups have higher average expenditures than both ICF groups ( $\$ 37.77$ per diem for SNF facilities compared to $\$ 29.12$ for ICF facilities; a significant difference at the 0.01 level). With regard to medical outcomes, an analysis of variance shows that $\mathrm{NF}$ group types are not significantly different from each other, and a $t$-test within each type (ICF or SNF) reveals no significant difference in the means between the two clusters in each type. This is observed in spite of conventional wisdom, which suggests that it is more difficult for SNF facilities to achieve a high level of medical outcomes due to the nature of their case mix relative to ICF facilities. However, this presumption does have indirect support given the difference in average per diems between the SNF and the ICF groups. That is, a more expensive case mix would imply a more expensive staff composition to manage, i.e., more registered nurses per resident.

There is however, a wide difference both between and within facility group types in terms of both satisfaction and social outcome ( $t$-tests of difference between means show that both satisfaction and social outcome are significantly higher in the SNF facilities than in the ICF facilities at the 0.05 level, that satisfaction and social outcomes are significantly higher in SNF group 2 than in SNF group 1 at the 0.01 level,

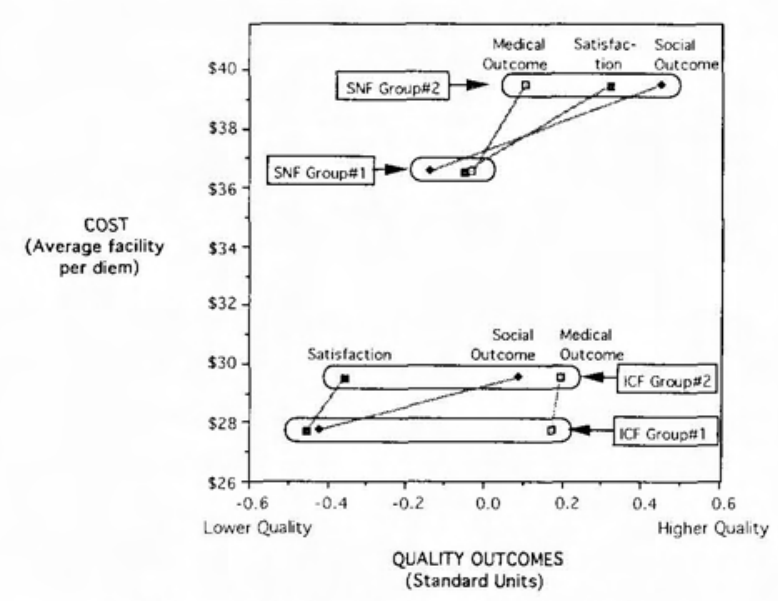

Fig. 4. Quality/Cost Relationships. 
that social outcome is significantly higher in ICF group 2 than in ICF group 1 at the 0.01 level, and that satisfaction is significantly higher in ICF group 2 than in ICF group 1 at the 0.10 level). ICF facilities appear to have significantly lower outcome quality scores on both satisfaction and social outcome than do SNF facilities. It appears that, as average facility per diem increases, social outcome and resident satisfaction increase. The question for TQM is, how do Figures 3 and 4 relate? From Figure 3, we observe that the dimension "facility" is a strong predictor of satisfaction (i.e., a strong negative path coefficient to violations, -0.219). Figure 3 also shows that facilities can choose to provide the resources, which together, promote an amenable environment, communication among residents and continuity of lifestyle; factors which promote long-term resident satisfaction. Facility and staffing characteristics are then indirectly and causally linked to changes in a resident's affective reaction (i.e., a resident's emotional perception of the care quality) to the overall care he/she receives.

\section{DISCUSSION}

The structure, process, and outcome taxonomy has long been used in the field of health care quality measurement and evaluation. However, it remains rare to see published, a true causal model which assesses facility level quality. One enormous advantage of being able to routinely assess facility or resident level quality in a causal framework is the ease with which it can become incorporated into a TQM program and CQI process. CQI is predicated upon the notion that managers can identify and articulate each causal linkage involved in the production of output, in this case nursing care. If CQI (and more broadly, TQM) is to succeed in health care, the ability to routinely assess quality and articulate causal linkages is vital. Furthermore, if heretofore controversial yet complex relationships to quality (i.e., quality and cost) are to be understood and managed proactively, causal assessment of quality is again necessary.

The current analysis demonstrates that nursing facilities may be substantially benefited by having access to causal linkages which materially affect outcome quality. Management would then have first-hand knowledge of structural characteristics and process activities that they may pursue in order to improve outcome quality. In addition, TQM posits that employee empowerment and "participative involvement of knowledgeable stakeholders" $(8$, p. 114) are required to initiate quality-improving actions, on the part of management. Similarly, Nyman (19) has argued that incentive-based policies (professional training, nonprofit status, desire to attract private pay patients, etc.), may more effectively improve nonhealth outcome quality than adequacy-based policies (policies designed to financially support some arbitrarily-defined minimum threshold of quality). Thus, if the facility knew the mechanism through which the affective portion of either process or outcome quality is influenced, they could manage that mechanism in order to inexpensively achieve improved outcomes.

Among various outcome measures which were investigated by Nyman (19), nonhealth outcomes (i.e., quality of life) were found to be significantly related to incentive-based policy and not to costs. Nonhealth quality measures are increasingly recognized as important to overall quality, and which can be inexpensively influenced by staff attitudes (19). Precisely how one sets out to improve a resident's quality of life is not known. However, some things are known regarding constituent elements of the quality of life construct (33). Establishing a protective, trusting, and respectful environment, residents feeling satisfied with staff attitudes and expertise, having a sense of justice and social participation, could all be components of an overall quality of life perception (34). In the current study, social outcome could be considered a component of a resident's quality of life. Note in Figure 3, that the staffing subdimension of structural quality positively influences social care, and that social care in turn, positively affects medical care and subsequently social outcome, which is an affective portion of overall outcome quality. Also, note that facility characteristics are directly and negatively related to "negative outcomes" (violations and complaints). Hence, it appears as though the professional credentials, numbers of professional staff, and total staff size can ultimately create staff attitudes, determine the variety and adequacy of resident activities, staff effectiveness, and care plan development as well as directly impact resident evaluation and resident influence in the process of care. Path analysis illustrates the causal mechanism by which such influence occurs, and makes directly accessible such a mechanism for management interested in employing the tenets of total quality management.

Whether the variation in social outcome and satisfaction between the SNF and ICF groups, shown in Figure 4 , is related to the differences in average per diem is difficult to address. Without rigorous case mix adjustment of direct care expenditures, for instance using the Resource Utilization Groups III algorithm (35), comparisons of quality related to expenditures are tenuous.

Applied to a large sample of nursing facilities with 
better data and case mix adjustment, the methodology could result in guidelines for continuous quality improvement and benchmarking or care quality standards. Applied continuously over time in one nursing facility, the methodology truly enables the effective implementation of continuous quality improvement and monitoring of care quality. The strength and direction of the relationships between structure, process, and outcome dimensions may help nursing facilities in prioritizing various potential interventions, and allow decision making to better follow the care objectives of each nursing home. For example, depending on the mix of patients, some nursing homes will emphasize health rehabilitation more than providing a high quality social environment, although all nursing homes would consider both objectives important and intertwined.

\section{CONCLUSION}

This study has presented a methodology to develop a causal model of the underlying relationships between structure, process, and outcome dimensions of quality of care in nursing facilities. The particular empirical test of this model presented in this paper is based on an instrument which measures quality of care in nursing facilities, the QAI. The methodology is useful in at least three ways. First, it provides a procedure for collecting and processing information aimed at continuous quality improvement in nursing facilities. Second, it provides quantitative estimates of the associations between key underlying factors that may serve as the basis for the actual design and implementation of quality improvement actions within nursing facilities. The causal model developed in this paper allows one to highlight the intensity as well as the direction of relationships between different variables related to the structure, process, and outcome of care delivered in nursing facilities. Finally, it provides directly useful implications for quality improvement strategies, and moves the field of quality in health care from a negative attitude of sanctioning to ensure minimal levels of quality, to a positive attitude of continuous, proactive improvement of quality which directly involves the staff.

We believe that this study opens an important research avenue. The methodology presented in this paper should be applied to better data and with superior case mix adjustment of direct care costs. Given the limitations of the data used for illustration in this paper, we believe that the methodology will benefit from data that are (a) collected routinely in each nursing facility; (b) collected at the resident level so as to allow for proper adjustment of the initial and current clinical condition of each resident; (c) collected on a large sample of nursing facilities that represent the different patterns of care in different market environments; and (d) collected over time.

The Minimum Data Set (36) would satisfy the above criteria. The Minimum Data Set is being developed under the auspices of the Omnibus Budget Reconciliation Act of 1987 to form a comprehensive and longitudinal data set on nursing home care. More thoroughly operationalizing quality with multiple indicators of structure, process, and outcome over time can be done with quality indicators (4) representing all care areas of nursing care. Such criteria are not new (37). The development of quality indicators has been called for by many researchers $(1,4,38,39)$. In fact, the use of quality indicators which both satisfy the above data criteria and which are specific to particular clinical conditions and care areas, would enormously enhance the reliability and validity of causal models using the structure, process, and outcome paradigm. Subsequently, better causal models would beget a better application of TQM to the nursing industry.

\section{References}

1. Davis M. On nursing home quality: a review and analysis. Med Care Rev 1991;48:129.

2. World Health Organization. Alma-Ata 1978. Primary Health Care. Report of the International Conference on Primary Health Care, Alma-Ata, USSR, 6-12 September 1978. Geneva, 1978.

3. Institute of Medicine. Improving The Quality of Care in Nursing Homes, , Washington D.C., National Academy Press, 1986

4. Gustafson DH, F Sainfort, R Van Koningsveld, et al. The quality assessment index (QAI) for measuring nursing home quality. Health Serv Res 25:1(April 1990):97-127.

5. Deming WE. Out of the Crisis. Cambridge, Massachusetts, Massachusetts Institute of Technology, Center for Advanced Engineering Study, 1986.

6. Donabedian A. Evaluating the quality of medical care. Milbank Q 1966;44:166-206.

7. Donabedian A. Explorations in Quality Assessment and Monitoring: the Definitions of Quality and Approaches to its Assessment, (Vol.1). Ann Arbor, Michigan: Health Administration Press, 1980.

8. Jones DJ, JT Ziegenfuss. The administrative and clinical rationale for the total organization approach to continuous improvement. Qual Assur Util Rev 1993;8:112.

9. Berwick DM. Continuous improvement as an ideal in health care. $N$ Engl J Med 1989;320:53-56.

10. Laffel G, D Blumenthal. The case for using industrial quality management science in health care organizations. JAMA 1989;262:2869-2873.

11. Donabedian A. A Guide to Medical Care Administration, Volume II: Medical Care Appraisal - Quality and Utilization. New York, The American Public Health Association, 1969.

12. Kane R, R Kane. Long Term Care: Principles Programs and Policies, NY, Springer Publishing, 1991.

13. Linn MW, L Gurel, BS Linn. Patient outcome as a measure of quality of nursing home care. Am J Public Health 1977;67:337.

14. Dennis LC, RE Burke, KM Garber. Quality Evaluation System: 
An Approach for Patient Assessment. J Long-Term Care Admin $1977 ; 28$.

15. Ullmann SG. The impact of quality on cost in the provision of long-term care. Inquiry 1985;22:293-302.

16. Moos RH, S Lemke. Multiphasic Environmental Assessment Procedure, Palo Alto, Social Ecology Laboratory, Veteran's Administration, 1984.

17. Nyman J. The effect of competition on nursing home expenditures under prospective reimbursement. Health Serv Res 1988;23:555-574.

18. Gertler P. A Latent-Variable Model of Quality Determination. $J$ Bus and Econ Stat 1988;6(1):97.

19. Nyman J. Improving the quality of nursing home outcomes: are adequacy or incentive-oriented policies more effective? Med Care 1988a;26:1158.

20. Indices of Quality in Long-Term Care: Research and Practice. New York, NY, The National League of Nursing, 1989.

21. Hannan M, J Freeman. The population ecology of organizations. Am J Soc 1977;32:929-964.

22. Jolliffe IT. Principal Component Analysis. New York, SpringerVerlag, 1986.

23. Tucker LR. Relations of factor score estimates to their use. Psychometrika 1971;36:427-436.

24. Kim JO, CW Mueller. Factor Analysis. Statistical Methods and Practical Issues, Newbury Park, Sage Publications, 1978.

25. Asher B. Causal Modeling, Beverly Hills, CA: Sage Publications, 1987.

26. Berry WD. Nonrecursive Causal Models, Beverly Hills: Sage Publications, 1984.

27. Davis JA. The Logic of Causal Order, Sage University Paper series on Quantitative Applications in the Social Sciences, 07055. Beverly Hills, Sage Publications, 1988.

28. Heise D. Causal Analysis, New York, John Wiley, 1975.

29. Davis JA. Analyzing contingency tables with linear flow graphs: D system. In D. Heise (ed.), Sociological Methodology, San Francisco, Jossey-Bass, 1975.

30. Taylor DG. Analyzing qualitative data. In P.H. Rossi, J.D. Wright, and A.B. Anderson (eds.) Handbook of Survey Research. New York, Academic Press, 1983.

31. Ishikawa K. Guide to Quality Control. White Plains, New York, Asian Productivity Organization, 1982.

32. Johnson RA, Wichern DW. Applied Multivariate Statistical Analysis, Englewood Cliffs, NJ, Prentice Hall, 1988.

33. Mike V, JD Hornquist. Quality of Life Research and Ethics of Evidence. Qual Life Res 1992;1:273-276.

34. Hornquist JD. The concept of quality of life. Scand J Soc Med 1982;10:57-61.

35. The Multistate Nursing Home Case Mix and Quality Demonstration - Description of the Resource Utilization Group, Version III (RUG III) System, version 3/7/92. A Working Document by the University of Michigan, Rensselaer Polytechnic Institute and The Circle, March 1992.

36. Morris J, C Hawes, B Fries, et al. Designing the National Resident Assessment Instrument for Nursing Homes, The Gerontologist 1990;30(3):293-307.

37. Lohr KN. Outcome measurement: concepts and questions. Inquiry 1988;25:37-50.

38. Phillips C. Developing a method of assessing quality of care in nursing homes, using key indicators and population norms." $J$ Aging Health 1991;3:407-422.

39. Zinn JS, WE Aaronson, MD Rosko. The use of standardized indicators as quality improvement tools: an application in Pennsylvania nursing homes. Am J Med Qual 1993;8:72-78. 